\title{
Scaling for lobe and cleft patterns in particle-laden gravity currents
}

\author{
A. Jackson, B. Turnbull, and R. Munro \\ Process and Environmental Research Division, Faculty of Engineering, University of Nottingham, Nottingham, UK
}

Correspondence to: A. Jackson (evxaj@nottingham.ac.uk)

Received: 15 November 2012 - Accepted: 10 January 2013 - Published: 18 February 2013

\begin{abstract}
Lobe and cleft patterns are frequently observed at the leading edge of gravity currents, including nonBoussinesq particle-laden currents such as powder snow avalanches. Despite the importance of the instability in driving air entrainment, little is known about its origin or the mechanisms behind its development. In this paper we seek to gain a better understanding of these mechanisms from a laboratory scale model of powder snow avalanches using lightweight granular material.

The instability mechanisms in these flows appear to be a combination of those found in both homogeneous Boussinesq gravity currents and unsuspended granular flows, with the size of the granular particles playing a central role in determining the wavelength of the lobe and cleft pattern. When scaled by particle diameter a relationship between the Froude number and the wavelength of the lobe and cleft pattern is found, where the wavelength increases monotonically with the Froude number.
\end{abstract}

\section{Introduction}

Both homogeneous particle-laden gravity currents and flows of unsuspended granular material typically feature characteristic three-dimensional wavy patterns at their leading edge (as illustrated by Fig. 5). An example of this instability can be found at the leading edge of a powder snow avalanche. Although this lobe-and-cleft-type structure contributes significantly to air entrainment (Simpson and Britter, 1979), and thus the dynamics of powder snow avalanches, little is known about the mechanisms that cause the formation of these patterns. Here we attempt to gain a better understanding of the instability in particle-laden flows by experimentally modelling powder snow avalanches on a laboratory scale. A powder snow avalanche is an inclined gravity current consisting of fully suspended, lightweight granular material (pow- der snow) which has much greater density than the ambient fluid (density ratio $\approx 10$ ), and exhibits characteristics of both homogeneous gravity currents and unsuspended granular flows. Several instability mechanisms have been identified in gravity currents and unsuspended granular flows that may also play a role in powder snow avalanches.

For homogenous Boussinesq gravity currents flowing along a horizontal surface a shifting pattern of lobes and clefts has been observed at the leading edge (Simpson, 1972; Neufeld, 2002; Härtel et al., 2000). Clefts merge together whilst new ones form when a lobe reaches a certain size, as a result the total number of lobes and clefts remains roughly constant. Linear-stability analysis and direct numerical simulations (Härtel et al., 2000) have shown that the stagnation point at the front of a gravity current is located below the nose. The formation of the lobe and cleft pattern is the result of a local instability at the leading edge of the front caused by the area of unstable stratification in the flow region between the nose and the stagnation point. In this paper we will henceforth refer to this instability as the classical lobe and cleft instability. For clarity it should be pointed out that we will refer to the wavy patterns observed at the front of the flows as lobe-and-cleft-type patterns, however the formation of these patterns is not necessarily caused by the classic lobe and cleft instability. There are several other mechanisms that could be causing the formation of a lobe-and-cleft-type pattern, which we shall now introduce.

In contrast to homogenous Boussinesq gravity currents, flows of dense granular material moving down an inclined plane exhibit different forms of wave-like patterns at the moving front (Nohguchi and Ozawa, 2008; Pouliquen et al., 1997). While it is possible that the classic lobe and cleft instability causes the initial fluctuations in the moving front, the aforementioned continuously shifting pattern of lobes and clefts is not present in these cases. Instead a more stable pattern develops with lobes developing into fingers that 
accelerate downslope ahead of the remainder of the flow. This pattern is similar in appearance to the instability seen in viscous fluids flowing down an incline (Huppert, 1982); however, in the latter case the instability is driven by surface tension, which is not present in a granular material.

Nohguchi and Ozawa (2008) hypothesise that pairs of vortices form between clefts in the front of a granular flow. These vortices rotate in a manner that reduces the air drag force at the moving front, causing the vortices to grow and deform the initial perturbation into a finger-like pattern. The existence of a velocity boundary layer at the moving front has been proposed, the thickness of which is assumed to be equal to the distance that a single particle travelling at the flow velocity can move against the drag force of the static air. The velocity boundary layer thickness $\delta_{v}$ is therefore defined as

$\delta_{v}=\frac{2 \rho_{\mathrm{p}} d}{3 C_{\mathrm{d}} \rho_{\mathrm{a}}}$,

where $\rho_{\mathrm{p}}$ and $\rho_{\mathrm{a}}$ are the densities of the particle and the surrounding air respectively, $d$ is the diameter of a single particle, and $C_{\mathrm{d}}$ is the drag coefficient. In order to maintain steady-state vortex motion the radius of each lobe would need to be twice the thickness of the velocity boundary layer. This then suggests that the size of the lobes will be related to the drag forces acting on the particles as drag force plays a key role in determining the velocity boundary layer thickness.

A further mechanism that leads to the formation of lobeand-cleft-type patterns is caused by polydispersity of the granular medium (Pouliquen et al., 1997). The high concentration of large particles located in the vicinity of a cleft leads to a local increase of the friction in these areas. The material thus locally slows down, amplifying the perturbation and leading to the formation of fingers.

Finally, the Rayleigh-Taylor instability is a fingering instability that occurs at an interface between two fluids of different density, for example a heavy fluid initially lying on top of light fluid in a gravitational field (Lord Rayleigh, 1900; Taylor, 1950). For inviscid fluids the interface will always be unstable, with the growth rate of the unstable modes increasing as their wavelengths decrease (Chandrasekhar, 1961).

This paper focuses on studying the development of lobeand-cleft-type patterns at the leading edge of a laboratory scale avalanche, using line releases of expanded polystyrene beads suspended in air. Following the findings of Nohguchi and Ozawa (2008), we assume the granular material is homogeneous with narrow size distribution (see Table 1), that there is minimal lateral spreading at the edges of the flow, negating the need for sidewalls, and that the base of the chute is flat. We present detailed measurements of the wavelength of the pattern found at the leading edge of the flow, and consider how the wavelength depends on the key experiment parameters.
Table 1. Properties of EPS beads used in our experiments.

\begin{tabular}{lcll}
\hline Diameter*, & Bulk Density, & $\begin{array}{l}\text { Particle } \\
\text { Reynolds } \\
\text { Number*, }\end{array}$ & $\begin{array}{l}\text { Settling } \\
\text { Velocity* }\end{array}$ \\
& & $\operatorname{Re}_{\mathrm{p}}$ & $v_{\mathrm{t}}\left(\mathrm{m} \mathrm{s}^{-1}\right)$ \\
\hline$(\mathrm{mm})$ & $\rho_{\mathrm{p}}\left(\mathrm{kg} \mathrm{m}^{-3}\right)$ & $175 \pm 15$ & $0.5 \pm 0.05$ \\
$1.3 \pm 0.05$ & 22 & $200 \pm 17$ & $0.6 \pm 0.06$ \\
$1.5 \pm 0.06$ & 22 & $225 \pm 22$ & $0.7 \pm 0.07$ \\
$1.8 \pm 0.09$ & 21 & $250 \pm 24$ & $0.7 \pm 0.07$ \\
$2.1 \pm 0.07$ & 21 & $325 \pm 28$ & $0.8 \pm 0.07$ \\
$2.5 \pm 0.09$ & 20 & $325 \pm 29$ & $0.8 \pm 0.09$ \\
$2.7 \pm 0.08$ & 19 & $525 \pm 51$ & $1.1 \pm 0.08$ \\
$5.0 \pm 0.13$ & 11 & $750 \pm 72$ & $1.1 \pm 0.09$ \\
$6.8 \pm 0.20$ & 10 & & \\
\hline
\end{tabular}

* Mean value \pm 1 standard deviation

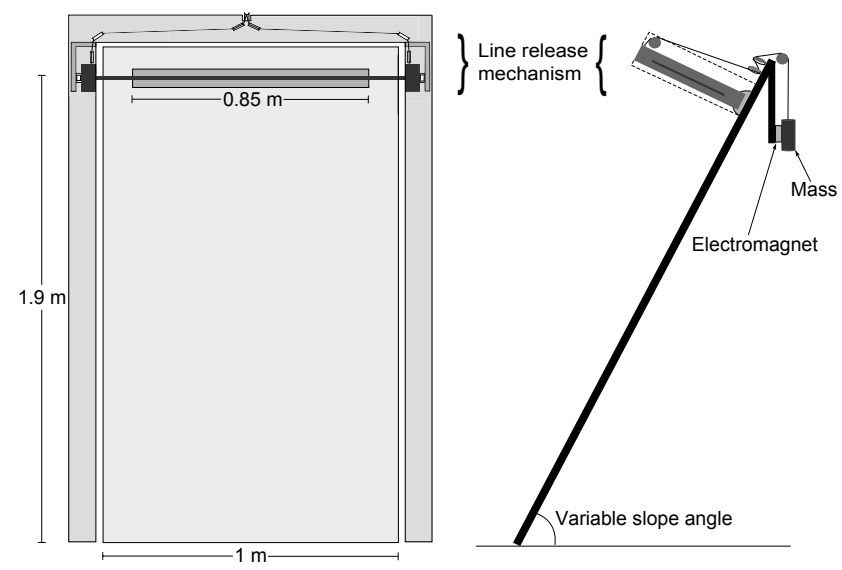

Fig. 1. Front and side view schematic of the chute used for experiments.

\section{Experiments}

The lightweight granular material used in this study is expanded-polystyrene (EPS) bead which has been shown (Turnbull and McElwaine, 2008) to provide a good model for powder-snow avalanches by matching important similarity criteria. The bulk density of the EPS bead is $\rho_{\mathrm{p}} \approx 20 \mathrm{~kg} \mathrm{~m}^{-3}$, with a relative density $\rho_{\mathrm{p}} / \rho_{\mathrm{a}} \approx 17$, where $\rho_{\mathrm{a}}=1.20 \mathrm{~kg} \mathrm{~m}^{-3}$ denotes air density (at $20^{\circ} \mathrm{C}$ and $1 \mathrm{~atm}$ ) and is here assumed throughout to be a constant. Eight different sizes of particles are used in the experiments, the properties of which are shown in Table 1. The relative density of the EPS beads means that the relatively high level of momentum carried by the snow particles in a powder-snow avalanche is well modelled.

The experiments were carried out using a $1 \mathrm{~m}$ wide, $1.9 \mathrm{~m}$ long, flat chute (Fig. 1). The chute was covered in a black felt cloth and inclined at slopes between $60^{\circ}$ and $90^{\circ}$, where $\theta$ denotes the angle between the chute and the horizontal. The EPS beads were released using an electromagnetically controlled hopper attached to the top of the chute (see Fig. 1). In 
order to create a pseudo two-dimensional line release, a linear hopper was used which had a semi-elliptical cross section (with aspect ratio $=0.9$ ) and a length of $0.85 \mathrm{~m}$. Three different sized linear hoppers were used so that the release volume could be varied between $1000 \mathrm{~cm}^{3}, 2100 \mathrm{~cm}^{3}$ and $3300 \mathrm{~cm}^{3}$. Each flow was observed using two high-speed digital video cameras sampling at $400 \mathrm{~Hz}$. One camera was positioned normal to the transverse plane of the chute to capture the plan view of the flow, ensuring that the full width of the release hopper and the full length of the chute were in view. The second camera was positioned to the side and aligned to view across the transverse plane of the chute, which allowed the head height of the avalanche to be measured. Schematic diagrams of a laboratory-scale avalanche, including the relevant parameters and variables, are shown in Fig. 2.

The particle Reynolds number for a bead of diameter $d$ moving with velocity $u$ is

$$
R e_{\mathrm{p}}=\frac{\rho_{\mathrm{a}} u d}{\mu}
$$

where $\mu$ is the dynamic viscosity of the ambient fluid. The particle Reynolds number determines whether the drag acting on the particle is dominated by viscous or pressure forces. Particle Reynolds numbers for powder snow avalanches are typically of the order $3 \times 10^{3}$ (Turnbull et al., 2007) and the drag force exerted on the particles from the ambient fluid will largely be due to the form drag of the particles. For values $500<R e_{\mathrm{p}}<10^{5}$ the drag coefficient for a spherical particle is essentially independent of the particle Reynolds number (Massey, 2006). Typical values of $R e_{\mathrm{p}}$ for the EPS beads used in the experiments reported are shown in Table 1, and lie on the lower boundary of this range, but correspond to the case where the pressure forces are dominant compared to the viscous forces.

The sedimentation velocity of a particle normal to the slope, $v_{\mathrm{t}}$, needs to be taken into account in order to ensure that suspension is maintained over the length of the chute. The length scale

$$
L=\frac{v_{\mathrm{t}}^{2}}{g}
$$

determines the size of the experiment. $v_{\mathrm{t}}$ was measured by releasing individual EPS beads with the chute inclined at $90^{\circ}$ to the horizontal. Using the captured images, the particles were tracked and their terminal velocity calculated. Typical values of $L$ for our experiments are $\approx 0.1 \mathrm{~m}$, much less than the vertical length of the chute, meaning suspension will be maintained and a steady-state powder snow avalanche will be well modelled.

The Richardson number for a layer of height $h$ and velocity $u$ on a slope at angle $\theta$ is

$R i=\frac{g^{\prime} h \cos \theta}{u^{2}}$,

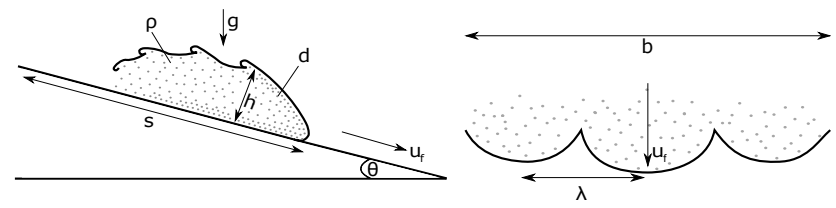

Fig. 2. Schematic diagrams of a particulate gravity current of height $h$, width $b$, density $\rho$ travelling a distance $s$ down an inclined plane with a front velocity $u_{\mathrm{f}}$. The particulate material has a diameter $d$ and a lobe and cleft pattern with wavelength $\lambda$ forms at the moving front.

where the reduced gravity $g^{\prime}=g\left(\rho-\rho_{\mathrm{a}}\right) / \rho_{\mathrm{a}}$ with $\rho$ the density of the current. The density ratio $\left(\rho-\rho_{\mathrm{a}}\right) / \rho_{\mathrm{a}}$ is typically used to calculate reduced gravity for fully suspended gravity currents (Turner, 1973) and is comparable to the Atwood number used for other types of density stratified flow. By using steep slope angles the values of $R i$ in our experiments $(R i \approx 2)$ were comparable to those observed in fullscale powder-snow avalanches $(R i \approx 1)$. In order to calculate $\rho$, the head of the current is assumed to be semi-circular in cross section, with radius equal to the current head height, $h$. The volume of the current can then be calculated as $V_{\mathrm{c}}=\left(\pi b h^{2}\right) / 2$ where $b$ is the width of the release hopper. The proportion of $V_{\mathrm{c}}$ that is made up of entrained air is then obtained by subtracting the initial release volume, $V_{\mathrm{i}}$ from $V_{\mathrm{c}}$. For the purpose of calculating $\rho$ it is also assumed that all of the EPS beads released are contained within the current head, meaning that the proportion of $V_{c}$ made up of EPS bead will be equal to $V_{\mathrm{i}}$. The density of the current is then calculated as $\rho=\left[(1-\alpha) \rho_{\mathrm{a}}+\alpha \rho_{\mathrm{p}}\right]$, where $\alpha=V_{\mathrm{i}} / V_{\mathrm{c}}$.

\section{Method for tracking the moving front and lobe and cleft formation}

The captured images were analysed using a level set method (McElwaine et al., 2004) to accurately track the front position of the flow and determine the formation of lobes and clefts. For each sequence of images the arrival time $\mathbf{T}(x, y)$ of the flow is detected for each pixel. The matrix $\mathbf{T}(x, y)$ of first arrival times is classified as a level set, with the contours $\mathbf{T}(x, y)=t$ corresponding to the front of the flow at a particular time. This technique was applied to both the plan view and side view images. The contours were used to measure flow properties, such as current head height, front position and velocity.

Calculating the gradient of the level set $(\nabla T)$ produces a vector field where each vector is normal to the surface of the moving front. The divergence of the vector field can be used to calculate the curvature of the level set,

$\kappa=\nabla \cdot \frac{\nabla T}{|\nabla T|}$.

In the next section we show how the curvature can be used to provide a robust and repeatable method for detecting and 
(a)

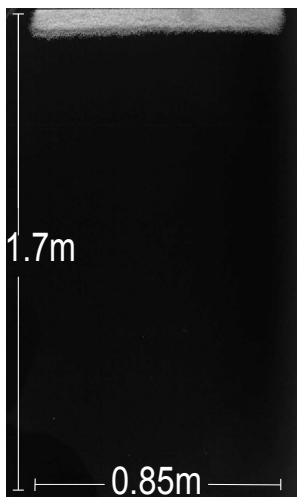

(b)

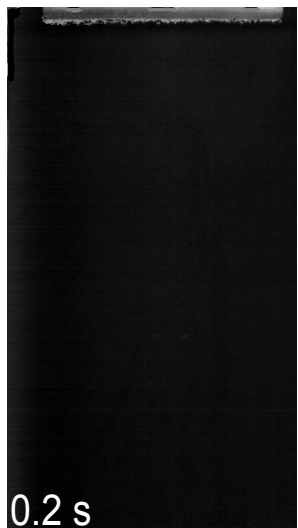

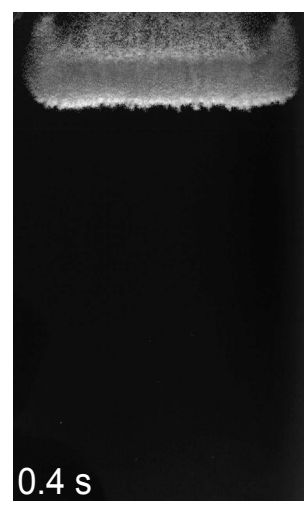
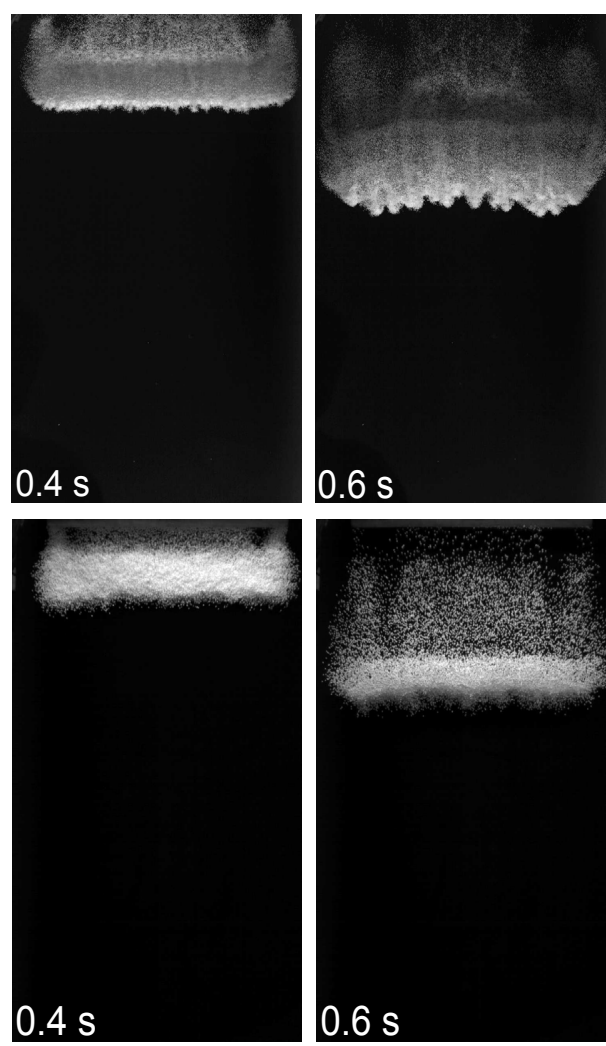
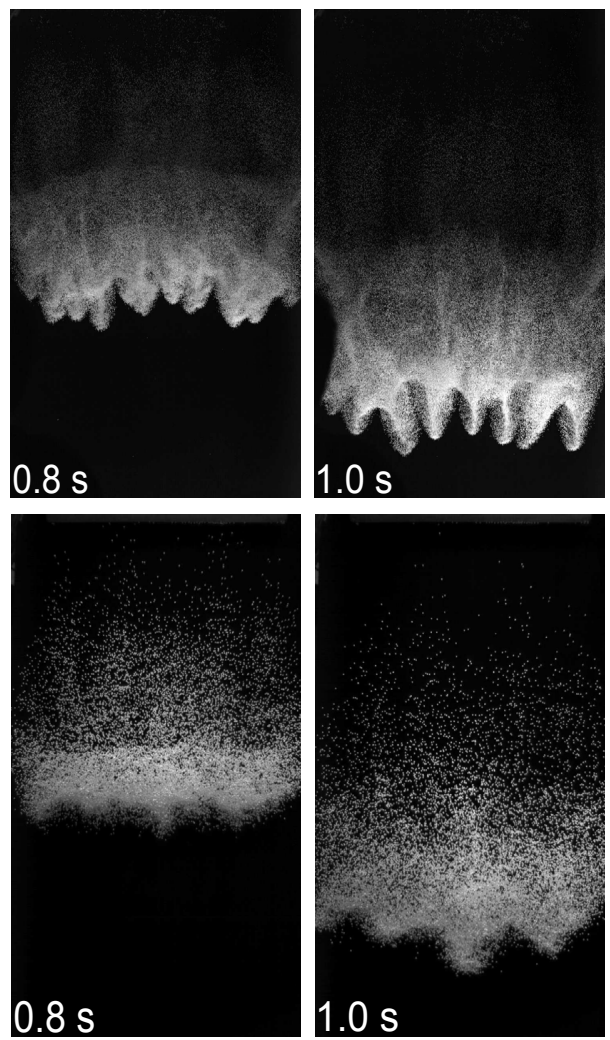

Fig. 3. Video stills taken at $0.2 \mathrm{~s}$ intervals from experiments conducted at a slope angle of $65^{\circ}$ using: (a) $2100 \mathrm{~cm}^{3}$ of $1.8 \mathrm{~mm}$ diameter EPS beads and (b) $3300 \mathrm{~cm}^{3}$ of $5.0 \mathrm{~mm}$ diameter EPS beads.

tracking lobes and clefts, with lobes corresponding to regions of small positive curvature and clefts to lines of large negative curvature.

\section{Results}

For each particle diameter, $d$, shown in Table 1 and for each initial release volume, $V_{\mathrm{i}}$, experiments were carried out at five different slope angles: $65^{\circ}, 70^{\circ}, 75^{\circ}, 80^{\circ}$ and $85^{\circ}$. Figure 3 shows images from two separate experiments: (a) for $d=1.8 \mathrm{~mm}$ and a release volume of $2100 \mathrm{~cm}^{3}$; and (b) for $d=5.0 \mathrm{~mm}$ and a release volume of $3300 \mathrm{~cm}^{3}$. In both cases the slope angle was set at $65^{\circ}$. In each experiment a welldefined front is observed that propagates down the slope. Immediately after the release the front is seen to accelerate down the slope for a short distance $(\approx 0.5 \mathrm{~m})$ before reaching an approximately constant velocity.

Although the particles start from a pseudo twodimensional linear distribution, a wavy pattern quickly appears in the moving front. As the fronts continue to propagate downslope these fluctuations grow in amplitude. Clear qualitative differences can be observed between the two flows shown in Fig. 3. One of the most striking is the difference in wavelength of the lobe-and-cleft-type pattern created by the fluctuations in the moving front during the later stages of the flows motion. That is, the lobe and cleft structure in Fig. 3a (for $d=1.8 \mathrm{~mm}$ ), has a noticeably smaller wavelength than that observed for the larger particles shown in Fig. 3b (for $d=5.0 \mathrm{~mm}$ ). Also, the lobes that form in Fig. 3a (for $d=1.8 \mathrm{~mm}$ ) have a greater amplitude than those in Fig. $3 \mathrm{~b}$ (for $d=5.0 \mathrm{~mm}$ ).

By taking particle diameter $d$ as our lengthscale, a nondimensional timescale can be calculated,

$\tilde{t}=t / \sqrt{d / g}$.

Images taken at the same non-dimensional time, $\tilde{t}=50$, from four experiments using the same release volume, but different sized particles are shown in Fig. 4. It can be shown that for a given value of $\tilde{t}$, as particle diameter increases the wavelength of the lobe-and-cleft-type pattern and the downslope position of the front also increase, but the ratio of the wavelength to amplitude remains approximately constant.

Front profiles generated from the flows in Fig. $3 a$ and $b$ are shown in Fig. 5, with the curvature of the corresponding level sets shown in Fig. 6. These figures show an initially shifting pattern of lobes and clefts. Lobes develop and grow in size, occasionally bifurcating, whilst clefts regularly merge together. This indicates that the lobe and cleft instability observed in fully suspended, Boussinesq gravity currents is dominant during the early phases of the flow. In the 

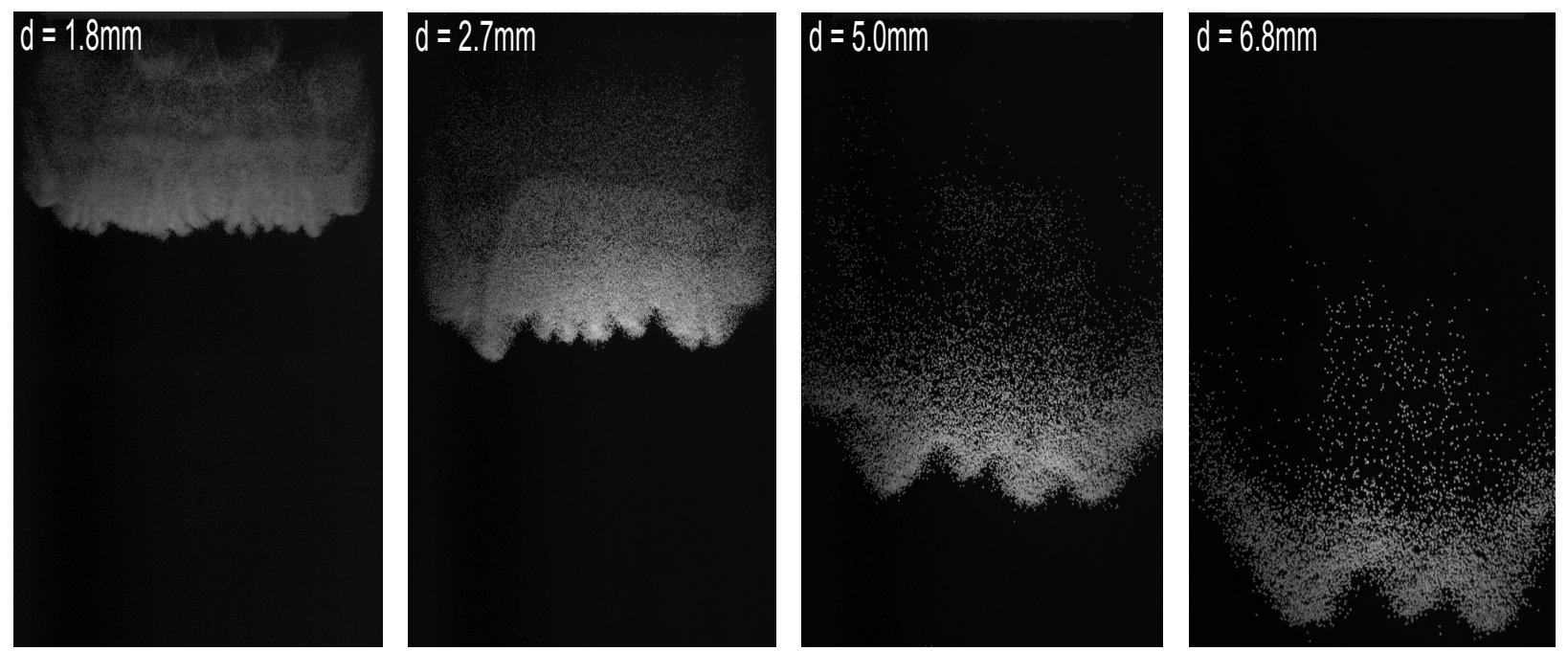

Fig. 4. Video stills taken at $\tilde{t}=50$ from experiments conducted at a slope angle of $65^{\circ}$ using $2100 \mathrm{~cm}^{3}$ of: $1.8 \mathrm{~mm}, 2.7 \mathrm{~mm}, 5.0 \mathrm{~mm}$ and $6.8 \mathrm{~mm}$ diameter EPS beads.

(a)

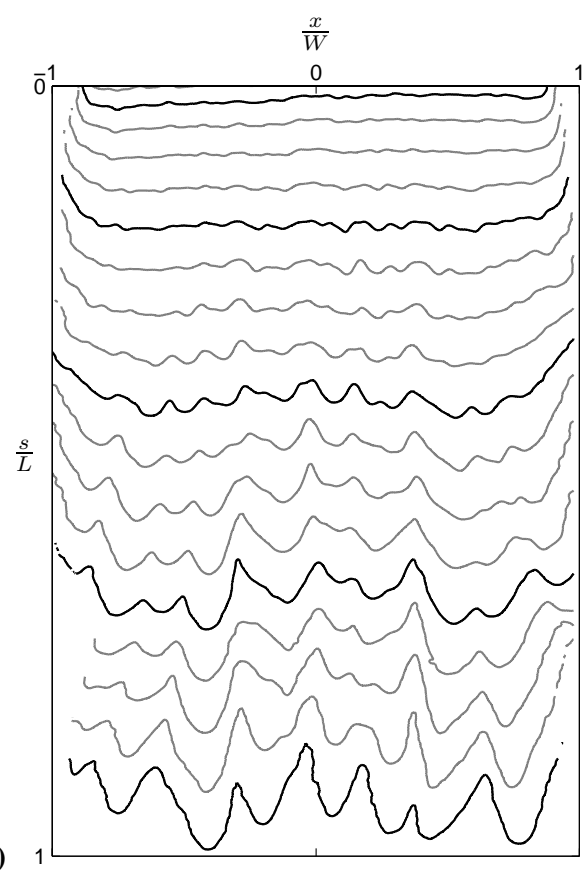

(b)

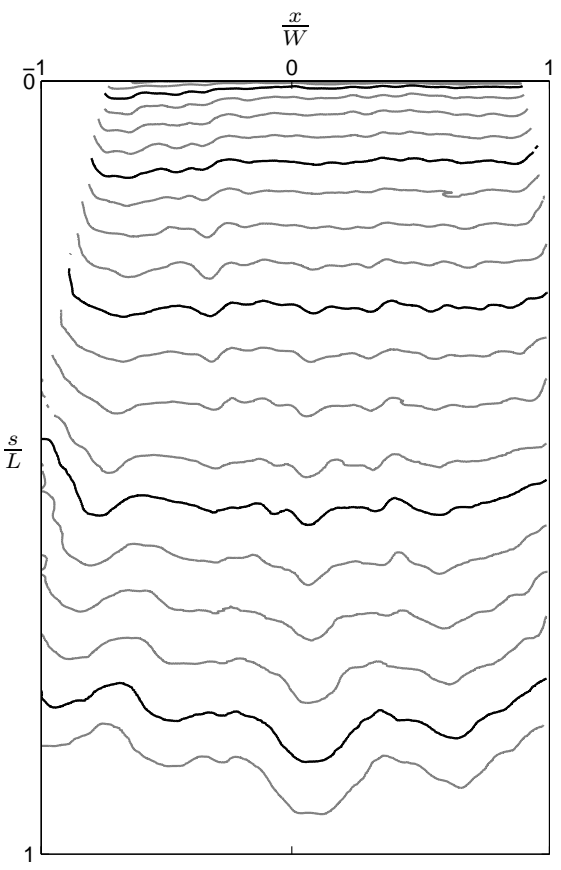

Fig. 5. (a) and (b) front profiles at $0.05 \mathrm{~s}$ intervals taken from the flows shown in Fig. $3 \mathrm{a}$ and b, respectively. The highlighted profiles correspond to the position of the fronts at the times shown in Fig. 3.

later stages of the flow, the position of the lobes is much more stable and there is less merging and bifurcation. As already noted, at later times the amplitude of the lobes has increased, this is particularly apparent for the flows consisting of smaller particles. This indicates that the fingering instabilities observed in unsuspended granular flows become more dominant in the later phases of the flow.

In order to investigate the effects of changing the release volume, wavelength against average distance from the line release $(s)$ for one particle size $(2.7 \mathrm{~mm})$ is shown in Fig. 7. Results from each of the three different release volumes are plotted separately and it can be seen that the volume of granular material released has no effect on the wavelength of the pattern in the moving front. Changing the volume of material released did have an observable effect towards the rear of the flows; as the volume was increased, the amount of detrainment at the rear of the flow also increased. 

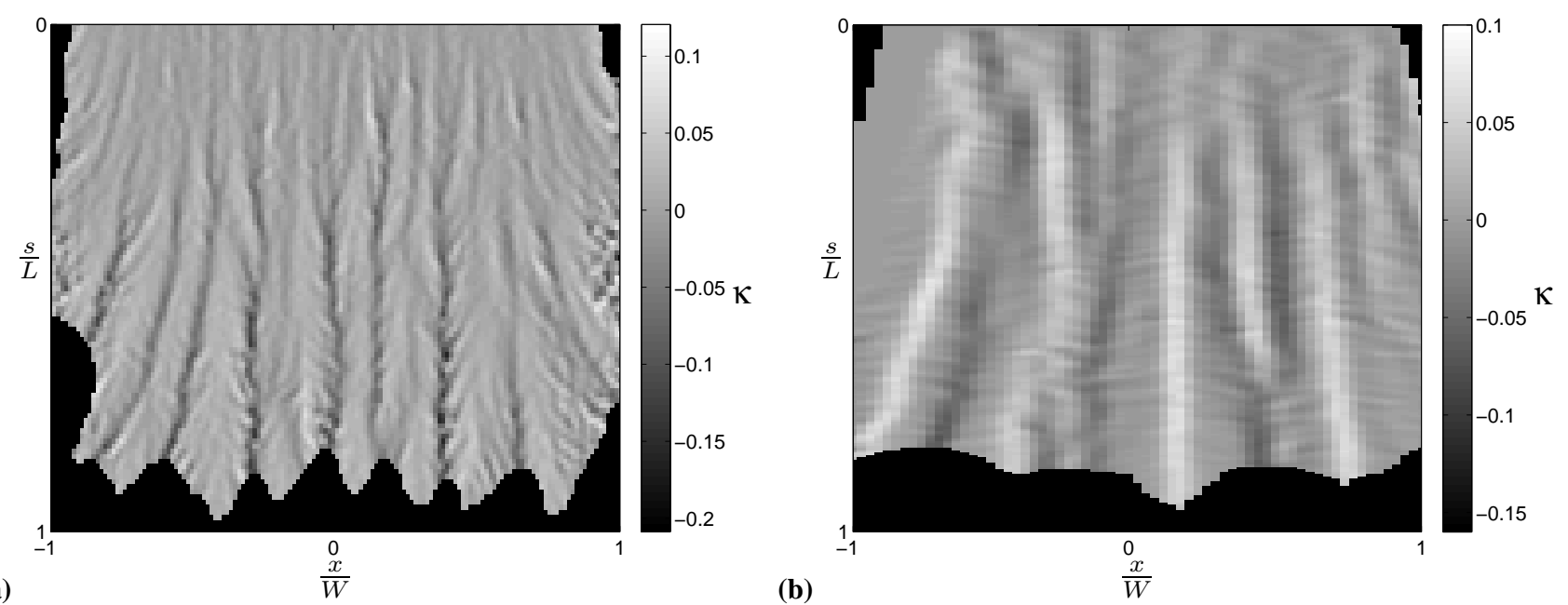

Fig. 6. (a) and (b) curvature $(\kappa)$ of the level set $\mathbf{T}(x, y)$ calculated from the flows shown in Fig. 3a and b, respectively. Areas of negative curvature (dark) indicate the location of clefts, whilst areas of positive curvature (light) indicate the location of lobes.

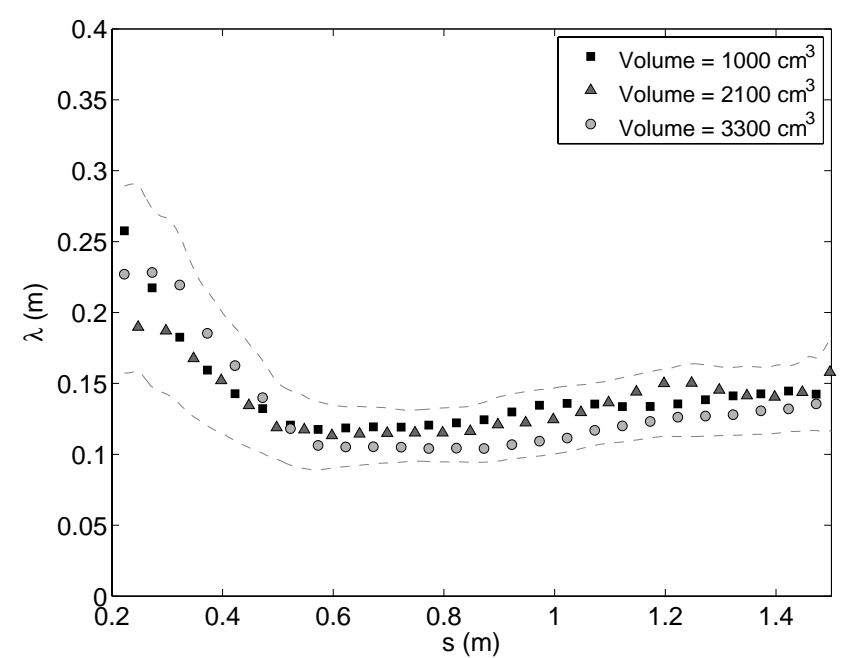

Fig. 7. Average wavelength $(\lambda)$ versus distance from line release $(s)$ for experiments using $2.7 \pm 0.08 \mathrm{~mm}$ diameter particles, plus or minus the standard deviation from the mean (dashed lines).

Figure 8 shows average wavelength $(\lambda)$ plotted against average distance from the line release for each particle size. The wavelength values for each particle size are obtained by taking the average from experiments conducted using the three release volumes. The results verify the observation that the pattern observed in the flows made up of smaller particles had a smaller wavelength than those made up of larger particles. It can also be seen that the wavelength remains relatively stable once the flow has reached an approximately constant velocity.

Current head heights for flows consisting of $1.8 \mathrm{~mm}$ and $6.8 \mathrm{~mm}$ diameter EPS beads are shown for each release volume and slope angle in Fig. 9. Several trends can be seen in the data; current height increases monotonically with in-

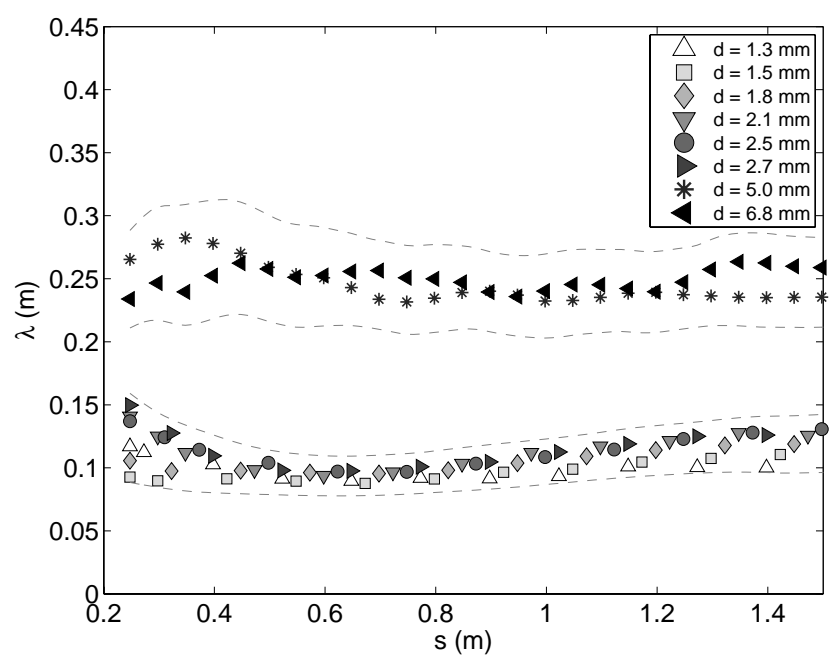

Fig. 8. Average wavelength $(\lambda)$ versus distance from line release $(s)$, plus or minus the standard deviation from the mean (dashed lines).

creasing slope angle and release volume, but with decreasing particle diameter. That is, currents consisting of smaller particles have larger heads, and therefore higher levels of suspension, due to the particles having lower settling velocities resulting in lower levels of particle sedimentation. Larger heads and greater levels of suspension are observed at higher slope angles as the component of the particles settling velocity normal to the slope is reduced. The data in Fig. 9 shows agreement with qualitative observations of detrainment levels at the rear of the flow. That is, it was observed that as the release volume was increased, higher levels of rear detrainment were observed, especially for currents consisting of smaller diameter EPS beads. Increased detrainment was also observed at higher slope angles. These observations are supported by Fig. 9 where the data for the smaller particles 


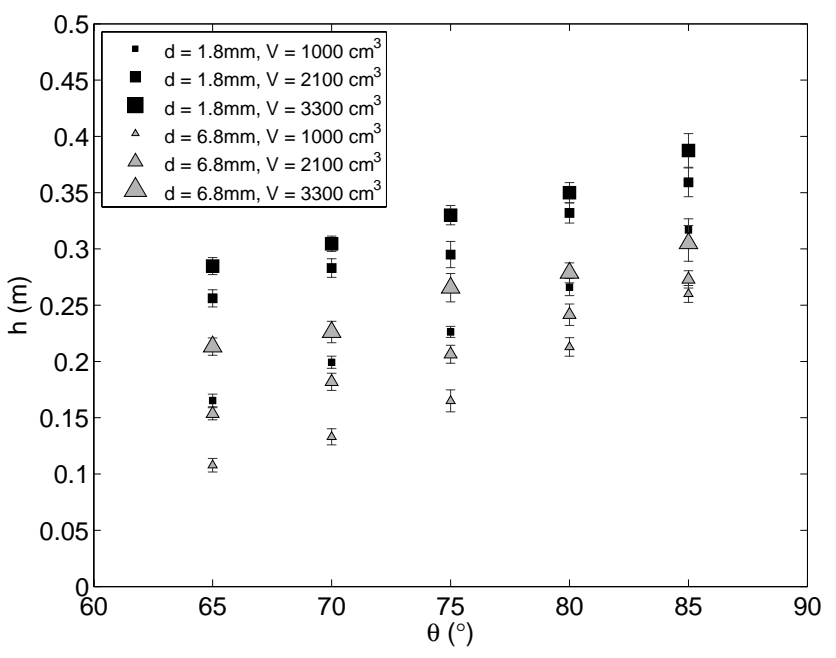

Fig. 9. Current head height $(h)$ versus slope angle $(\theta)$ for currents consisting of $1.8 \mathrm{~mm}$ and $6.8 \mathrm{~mm}$ diameter EPS beads. Head height was measured at a distance of $1 \mathrm{~m}$ from the line release. Error bars have length equal to the standard deviation from the mean.

shows a much smaller increase in current head height between the middle and largest size of release volume than that between the smallest and middle size. For both particle sizes the difference in current head height between the three release volume sizes decreases as slope angle increases. These observations and results indicate that there may be a limit to the amount of material that can be suspended within the head of the current, and as this limit is approached the amount of material detrained from the rear of the head increases.

In Fig. 10 the average wavelength at a distance of $1 \mathrm{~m}$ from the line release is plotted against slope angle for each of the particle sizes used. It was observed during the experiments that increasing the angle of the slope increased the level of suspension within the flow. At higher slope angles, more air was entrained into the current and its height $(h)$ perpendicular to the slope visibly increased. As the slope angle becomes steeper, the increased component of gravity driving the current is counteracted by an increase in air entrainment (Turnbull and McElwaine, 2007). However, it can be seen from Fig. 10 that the wavelength of the pattern is independent of the slope angle, indicating that level of suspension or air entrainment has no effect on the formation of the pattern. Interestingly, in Fig. 10 there appears to be a bifurcation in the data between the smaller particles $(d=1.5-2.7 \mathrm{~mm})$ and the larger particles $(d=5.0-6.8 \mathrm{~mm})$. This bifurcation could be due to the higher levels of polydispersity of the smaller particles (standard deviation $\approx 5 \%$ of mean particle diameter) compared with the larger particles (standard deviation $\approx 2.5 \%$ of mean particle diameter). Whilst it is possible that the granular fingering mechanism observed by Pouliquen et al. (1997) could play a more significant role in the flows consisting of smaller particles, this seems unlikely as in the experiments conducted by Pouliquen et al. this

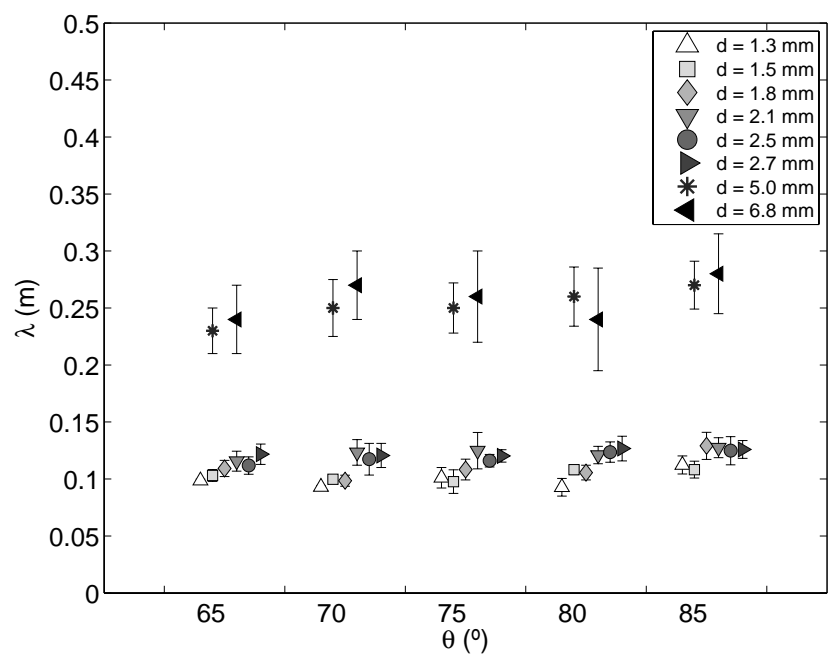

Fig. 10. Average wavelength $(\lambda) 1 \mathrm{~m}$ from the line release versus slope angle $(\theta)$. Error bars have length equal to the standard deviation from the mean. Results are offset horizontally for clarity.

mechanism was only observed when the standard deviation of the mean particle diameter was greater than $10 \%$. Another potential explanation for the bifurcation in the data could be the role played by viscous drag forces for the different particle sizes. As previously mentioned, the values of $R e_{\mathrm{p}}$ are high enough for pressure drag forces to constitute the bulk of the total drag force. However, whilst the larger particle's $R e_{\mathrm{p}}$ values are within the region where the drag coefficient for a spherical particle is independent of $R e_{\mathrm{p}}$, the smaller particle's values lie just below this region. Therefore, although pressure drag forces are still dominant, viscous forces will have a more significant effect on the drag force acting on the particles.

The Rayleigh-Taylor instability should be the limiting case as we tend towards a vertical chute. Hydrodynamically, the growth of the Rayleigh-Taylor instability is predicted by a perturbation analysis that shows that the growth rate of the fingers depends on the perturbation wavelength (Chandrasekhar, 1961; Youngs, 1984; Duff et al., 1962; Sharp, 1984). In the presence of a stabilising influence, e.g. viscosity or surface tension, some wavelengths are damped and a dominant mode develops. In our EPS suspension experiments, we have neither viscosity nor surface tension present. However, we speculate that the air drag experienced by the particle clusters, and the restrictions on lobe shape that this imposes (Bush et al., 2003), would play a similar role in allowing a dominant wavelength to grow. This should provide a rich focus for future modelling.

For comparison with the results of Nohguchi and Ozawa (2008), the mean radius of curvature of the lobes for each particle size was calculated (Fig. 11). These values were calculated from the level set contours corresponding to when the flows had reached a distance of $1 \mathrm{~m}$ down the chute ensuring 


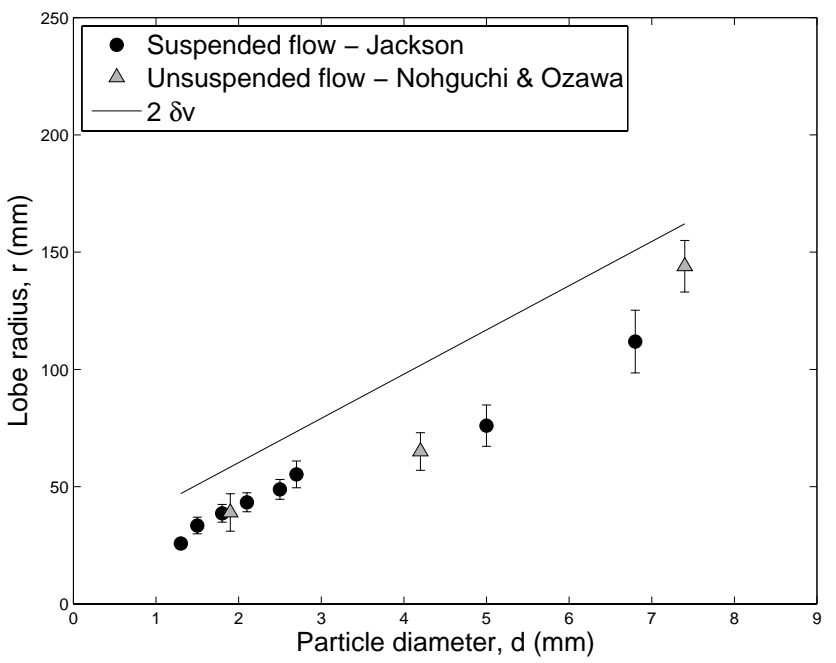

Fig. 11. Radius of curvature of lobes versus particle diameter. Results from the experiments presented in this paper $(\bullet)$ are compared with the experimental results $(\triangle)$ and analytical results (solid line) of Nohguchi and Ozawa (2008).

that, as with the results of Nohguchi and Ozawa (2008), the flows had reached an approximately steady velocity. The lobes at the leading edge of the flows consisting of smaller particles had smaller radii of curvature, consistent with the observations of sharper lobes and smaller wavelengths for these flows. The results show good agreement with Nohguchi and Ozawa's experimental data (which are also shown in Fig. 11), however both sets of data are slightly below the analytical estimate $r \approx 2 \delta_{v}$. When deriving the expression for boundary layer thickness Nohguchi and Ozawa ignored interparticle collisions in the velocity boundary layer for simplification. In reality, a particle moving through the velocity boundary layer is likely to experience collisions with other particles, reducing the distance that it can move against the drag force of the ambient fluid. Therefore, the analytical results are likely to overestimate the velocity boundary layer thickness which may explain the discrepancy seen between the theoretical and experimental results.

\section{Non-dimensionalisation}

Both granular fingering (Pouliquen et al., 1997) and velocity boundary layer (Nohguchi and Ozawa, 2008) theories suggest that the velocity of the moving front could play a role in determining the wavelength of the pattern that forms at the front. Fingers are created due to differences in velocity between smaller particles and larger particles that slow down due to higher levels of friction as they bunch up around clefts. A velocity boundary layer's thickness will also be affected by front velocity, which could in turn affect the wavelength of the pattern.
Using the variables shown in the schematic (Fig. 2), the non- dimensional group, which is the Froude number

$F r=\frac{u_{\mathrm{f}}}{\sqrt{g^{\prime} L \sin \theta}}$,

can be formed where $L$ is an appropriate length scale. The theory of a velocity boundary layer at the moving front suggests that there is a relationship between front velocity and wavelength which is determined by an individual particle's interaction with the ambient air. As previously mentioned, Nohguchi and Ozawa (2008) have proposed that the wavelength of the frontal pattern is determined by the size of pairs of vortices that form at the moving front. The size of these vortices is in turn determined by the thickness of the velocity boundary layer. Faster moving flows will have thicker velocity boundary layers as the particles can move a greater distance against the drag force of the static air, resulting in the formation of larger vortices and therefore a larger wavelength pattern. From Eq. (1) it can be seen that particle diameter $d$ is a key variable in determining the boundary layer thickness $\delta_{v}$; therefore, we will look for a relationship between the Froude number and wavelength based on the length scale $L=d$.

The dimensionless wavelength $\left(\tilde{\lambda}_{\mathrm{d}}=\lambda / d\right)$ is plotted against the Froude number in Fig. 12. Interestingly, when scaled with a length scale characterised by the particle diameter there appears to be a monotonically increasing relationship between the Froude number and wavelength. A linear fit of the data,

$\tilde{\lambda}_{\mathrm{d}}=2.3 F r_{\mathrm{d}}+1$,

is shown in Fig. 12, with a residual of 0.12 indicating a good level of fit.

This dimensionless rendering of the data seems to offer good agreement with the concept of a velocity boundary layer determining the wavelength of the frontal pattern. The data shows that when scaled with respect to particle diameter, the smaller particles have higher velocities and therefore thicker boundary layers resulting in wider lobes and a larger wavelength.

\section{Conclusions}

This paper has presented experiments on the flows of fully suspended, lightweight granular material down an inclined surface. These currents have been found to exhibit characteristics of the instabilities found at the leading edge of both homogenous particle-laden gravity currents and flows of unsuspended granular material. During the early stages of motion a lobe and cleft instability mechanism, similar to that observed in homogenous particle-laden gravity currents, appears to be dominant. As the flow propagates down the slope the pattern of lobes and clefts becomes more stable 


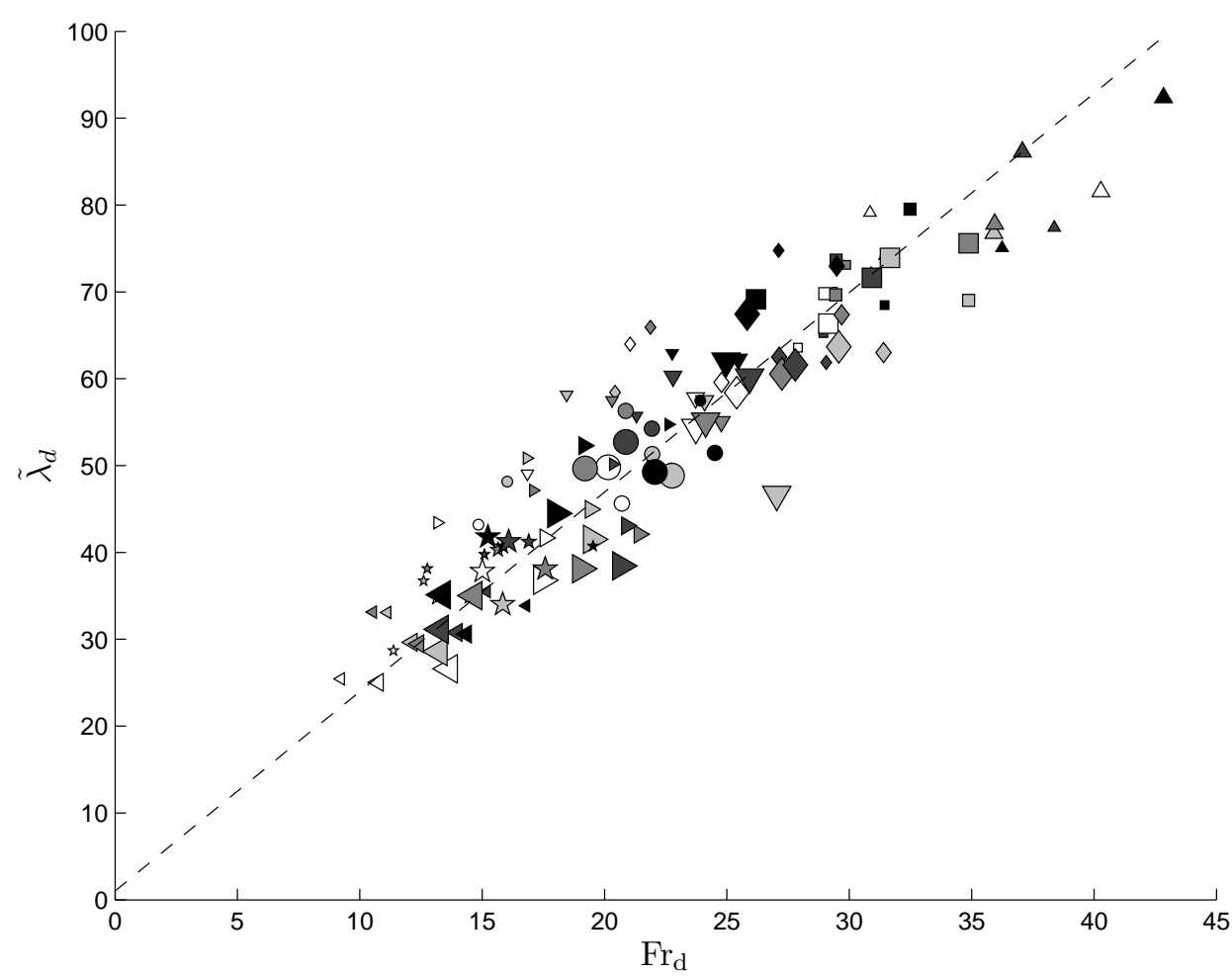

Fig. 12. Non-dimensional wavelength $\left(\tilde{\lambda}_{\mathrm{d}}\right)$ versus the Froude number $\left(F r_{\mathrm{d}}\right)$. Particle sizes: $1.3 \mathrm{~mm}(\triangle), 1.5 \mathrm{~mm}(\square), 1.8 \mathrm{~mm}(\diamond), 2.1 \mathrm{~mm}$ $(\nabla), 2.5 \mathrm{~mm}(\circ), 2.7 \mathrm{~mm}(\triangleright), 5.0 \mathrm{~mm}(\star)$ and $6.8 \mathrm{~mm}(\triangleleft)$. Marker shades correspond to slope angle: $65^{\circ}-$ clear, through to $85^{\circ}-$ black. Marker size corresponds to the size of the release volume. Dashed line shows linear fit, $\tilde{\lambda}_{\mathrm{d}}=2.3 \mathcal{F} r_{\mathrm{d}}+1$ (Eq. 8).

suggesting that the dominant instability mechanism is now more similar to those observed in unsuspended granular flows. Particle diameter of the granular material has been found to play a central role in determining the wavelength of the lobe and cleft pattern, as hypothesised by Nohguchi and Ozawa (2008). When scaled with particle diameter, a relationship between the Froude number of the flow and wavelength of the lobe and cleft pattern has been found. This relationship highlights the importance of the role played by drag forces acting on the individual particles in determining the wavelength of the frontal pattern, and appears to support the concept of a velocity boundary layer at the moving front of the flow.

Edited by: J. M. Redondo

Reviewed by: J. McElwaine and one anonymous referee

\section{References}

Bush, J., Thurber, B., and F., B.: Particle clouds in homogeneous and stratified environments, J. Fluid Mech., 489, 29-54, doi:10.1017/S0022112003005160, 2003.

Chandrasekhar, S.: Hydrodynamic and Hydromagnetic Stabiltiy, Oxford University Press, Oxford, UK, 1961.
Duff, R., Harlow, F., and Hirt, C.: Effects of diffusion on interface instability between gases, Phys. Fluids, 5, 417-425, 1962.

Härtel, C., Carlsson, F., and Thunblom, M.: Analysis and direct numerical simulation of the flow at a gravity-current head. Part 2. The lobe-and-cleft instability, J. Fluid Mech., 418, 213-229, 2000.

Huppert, H.: Flow and instability of a viscous current down a slope, Nature, 300, 427-9, 1982.

Lord Rayleigh: Investigation of the character of the equilibrium of an incompressible heavy fluid of variable density, in: Scientific Papers, vol. II, p. 200, Cambridge University Press, Cambridge, UK, 1900.

Massey, B.: Mechanics Of Fluids, Taylor \& Francis, Abingdon, UK, 8th Edn., 2006.

McElwaine, J., Patterson, M., and Dalziel, S.: Lobe and cleft formation at the head of a gravity current, Proceedings of the XXI International Congress of Theoretical and Applied Mechanics, 15-21, 2004.

Neufeld, J.: Lobe-cleft patterns in the leading edge of a gravity current, M.S. thesis, Department of Physics, University of Toronto, Canada, 2002.

Nohguchi, Y. and Ozawa, H.: On the vortex formation at the moving front of lightweight granular particles, Physica D, 238, 20-26, doi:10.1016/j.physd.2008.08.019, 2008.

Pouliquen, O., Delour, J., and Savage, S.: Fingering in granular flows, Nature, 386, 816-817, 1997.

Sharp, D.: An overview of Rayleigh-Taylor instability, Physica D, 12, 3-18, 1984. 
Simpson, J.: Effects of the lower boundary on the head of a gravity current, J. Fluid Mech., 53, 759-768, 1972.

Simpson, J. and Britter, R.: The dynamics of the head of a gravity current advancing over a horizontal surface, J. Fluid Mech., 94, 477-495, 1979.

Taylor, G.: The instability of liquid surfaces when accelerated in a direction perpendicular to their planes, I, Proc. R. Soc. Lon. Ser. A, 201, 192-196, 1950.

Turnbull, B. and McElwaine, J.: A comparison of powder-snow avalnches at Vallee de la Sionne, Switzerland, with plume theories, J. Glaciol., 53, 30-40, 2007.
Turnbull, B. and McElwaine, J.: Experiments on the nonBoussineq Flow of Self-Igniting Suspension Currents on a Steep Open Slope, J. Geophys. Res., 113, F01003, doi:10.1029/2007JF000753, 2008.

Turnbull, B., McElwaine, J., and Ancey, C.: KulikovskiySveshnikova-Beghin model of powder snow avalanches: Development and application, J. Geophys. Res., 112, F01004, doi:10.1029/2006JF000489, 2007.

Turner, J.: Buoyancy Effects in Fluids, Cambridge University Press, Cambridge, UK, 1973.

Youngs, D.: Numerical simulation of turbulent mixing by RayleighTaylor instability, Physica D, 12, 32-34, 1984. 